\title{
The prognostic significance of global aberrant alternative splicing in patients with myelodysplastic syndrome
}

\author{
Yi-Tsung Yang ${ }^{1,2}$, Yu-Chiao Chiu $\mathbb{0}^{3}$, Chein-Jun Kao ${ }^{4}$, Hsin-An Hou ${ }^{4}$, Chien-Chin Lin ${ }^{4,5}$, Cheng-Hong Tsai $\mathbb{C}^{4,6}$, \\ Mei-Hsuan Tseng ${ }^{4}$, Wen-Chien Chou ${ }^{4,5}$ and Hwei-Fang Tien ${ }^{4}$
}

\begin{abstract}
Aberrant alternative splicing (AS) is a hallmark of cancer development. However, there are limited data regarding its clinical implications in myelodysplastic syndrome (MDS). In this study, we performed an in-depth analysis of global AS in 176 primary MDS patients with 20 normal marrow transplant donors as reference. We found that $26.9 \%$ of the expressed genes genome-wide were aberrantly spliced in MDS patients compared with normal donors. These aberrant AS genes were related to pathways involved in cell proliferation, cell adhesion and protein degradation. A higher degree of global aberrant AS was associated with male gender and U2AF1 mutation, and predicted shorter overall survival and time to leukemic change. Moreover, it was an independent unfavorable prognostic factor irrespective of age, revised international prognostic scoring system (IPSS-R) risk, and mutations in SRSF2, ZRSR2, ASXL1, TP53, and EZH2. With LASSO-Cox regression method, we constructed a simple prognosis prediction model composed of 13 aberrant AS genes, and demonstrated that it could well stratify MDS patients into distinct risk groups. To our knowledge, this is the first report demonstrating significant prognostic impacts of aberrant splicing on MDS patients. Further prospective studies in larger cohorts are needed to confirm our observations.
\end{abstract}

\section{Introduction}

Alternative splicing (AS) is a physiological phenomenon to ensure higher protein diversity for better environmental fit. Around $70 \%$ of human genes undergo AS and 95\% of multi-exonic genes are alternatively spliced ${ }^{1}$. Splicing is a complex and tightly regulated process. Perturbation of this process by spliceosome gene mutations, epigenetic modifications, or other causes would lead to aberrant AS, resulting in deregulation of many cellular processes, such as cell proliferation, adhesion, differentiation, motility, invasion, and death ${ }^{2,3}$.

Correspondence: W.-C. Chou (wchou@ntu.edu.tw) or

H.-F. Tien (hftien@ntu.edu.tw)

${ }^{1}$ Division of Hematology-Oncology, Department of Internal Medicine, National Taiwan University Hospital Hsin-Chu Branch, Hsinchu, Taiwan

${ }^{2}$ Graduate Institute of Clinical Medicine, College of Medicine, National Taiwan University, Taipei, Taiwan

Full list of author information is available at the end of the article.
Evidence has shown that aberrant AS is a hallmark of cancer development ${ }^{4}$. A recent study demonstrated that approximately $29 \%$ of the expressed genes genome-wide were aberrantly spliced in acute myeloid leukemia (AML) patients compared with normal donors ${ }^{5}$. The genes with aberrant AS encode several oncoproteins, tumor suppressor proteins, ribonucleoproteins, and proteins involved in apoptosis, cell proliferation, and spliceosome assembly ${ }^{5,6}$. These findings provide a link between aberrant AS and AML pathogenesis.

Myelodysplastic syndrome (MDS) is a highly heterogeneous disease whose pathogenesis is still under investigation. The pattern of global aberrant AS and its clinical implications in MDS are not well known yet. We wonder if aberrant AS would be related to the pathogenesis and clinical features of MDS. To answer these questions, we determined the global gene expression and splicing patterns from a large cohort of de novo MDS patients, using

\section{(c) The Author(s) 2018}

(c) Open Access This article is licensed under a Creative Commons Attribution 4.0 International License, which permits use, sharing, adaptation, distribution and reproduction cc) in any medium or format, as long as you give appropriate credit to the original author(s) and the source, provide a link to the Creative Commons license, and indicate if changes were made. The images or other third party material in this article are included in the article's Creative Commons license, unless indicated otherwise in a credit line to the material. If material is not included in the article's Creative Commons license and your intended use is not permitted by statutory regulation or exceeds the permitted use, you will need to obtain permission directly from the copyright holder. To view a copy of this license, visit http://creativecommons.org/licenses/by/4.0/. 
normal transplant donors as reference. With complete clinical and genetic data of these patients, we were able to analyze the clinical and pathological implications of global aberrant AS for MDS patients.

\section{Materials and methods Study design and patient cohort}

From November 1991 to December 2010, a total of 176 primary MDS patients who were diagnosed at the National Taiwan University Hospital (NTUH) and had available samples and complete clinical and gene mutation data were enrolled in this study. Twenty healthy bone marrow (BM) donors of hematopoietic stem cell transplantation (HSCT) in this period were collected as normal controls. We also recruited another cohort of 31 primary MDS patients diagnosed between January 2011 and May 2012 as a validation cohort to confirm the prognostic impact of aberrant AS. The patients were reclassified according to the 2016 World Health Organization (WHO) classification ${ }^{7}$. All patients gave their informed consent to participate in the study. This study was approved by the Institutional Review Board of the NTUH.

\section{Cytogenetic and mutation analyses}

Chromosome abnormalities ${ }^{8}$ and genetic mutation analyses were performed as previously reported ${ }^{9-18}$.

\section{Affymetrix HTA 2.0 analysis}

The RNA was extracted from BM mononuclear cells (BMMNCs) by the TRIzol method at initial MDS diagnosis. RNA concentration and integrity were evaluated with ND-1000 spectrophotometer (NanoDrop Technologies, Wilmington, DE, USA) and 2100 Bioanalyzer (Agilent Technologies, Palo Alto, CA, USA). One microgram of RNA from each sample was processed with the miRNeasy mini kit (Qiagen, Hilden, Germany) and then it was labeled with biotin, followed by hybridization to Affymetrix GeneChip Human Transcriptome Array 2.0 (HTA 2.0) (Affymetrix, Santa Clara, CA), and finally scanned at Microarray Core Lab of the National Health Research Institutes according to Affymetrix protocols. We transformed the intensities to digital signals by Affymetrix AGCC software for further analyses with the Affymetrix Expression Console software and Transcriptome Analysis Console (TAC) version 3.0 software (flowchart in Supplementary Figure S1). The raw and normalized microarray data of Affymetrix HTA 2.0 reported in this article have been deposited in the Gene Expression Omnibus database (accession number GSE107400).

\section{Definition of the parameters for aberrant splicing}

We used the splicing index (SI) method based on default setting of TAC version 3.0 software to define the aberrant AS events of MDS patients with normal control donors as reference ${ }^{19,20}$. A probe selection region (PSR) is a region with clustering probes in an exon. The SI of a PSR is derived from the ratio of the PSR signal intensity between MDS patients and normal control donors after normalization by the gene expression levels, i.e.,

$$
\begin{aligned}
& \text { Splicing index of RSRa } \\
& =\frac{\left[\frac{\text { Intensity of PSRa in gene A }}{\text { gene A expression level }}\right] \text { of MDS patient }}{\left[\frac{\text { Intensity of PSRa in gene A A }}{\text { gene A expression level }}\right] \text { of normal donor }}
\end{aligned}
$$

When the value is less than 1 , SI is defined as the negative reciprocal. For example, an SI value of $1 / 4$ is converted to -4 . SI has to be $<-2$ or $>2$ to be denoted as an aberrant AS event. Each gene may have more than one aberrant AS event, depending on how many PSRs with abnormal SI in that gene. A gene with at least one aberrant AS event is called an aberrant AS gene.

Next, we defined aberrant AS score as genome-wide total aberrant AS events divided by total aberrant AS genes in each MDS patient. Therefore, a higher aberrant AS score indicated more aberrant AS events in each aberrant AS gene on average, which implied a higher degree of aberrant AS.

\section{Bioinformatics analysis}

Functional annotation for the aberrant AS genes of whole genome was performed by the Database for Annotation, Visualization and Integrated Discovery (DAVID) web tool with default settings to comprehensively explore the dysregulated functional relevance of these genes.

We sought to construct a concise prognosis prediction model out of the aberrant AS events. All analyzable SI values of PSRs were log2 transformed and analyzed for the association with overall survival (OS) by the univariate Cox proportional hazards model. The prognostic aberrant AS events identified were then merged into a Least Absolute Shrinkage and Selection Operator (LASSO) analysis in the Cox model (LASSO-Cox) ${ }^{21}$. LASSO-Cox is a regression method that selects a subset of variables (i.e., aberrant AS events) to yield the best prediction of OS while reducing overfitting of data. Here the optimal subset of aberrant AS events was determined by a leave-one-out cross validation analysis within the training cohort. As a result, LASSO-Cox assigned a regression coefficient to each of the selected aberrant AS events. Based on the results, we constructed a scoring system by a weighted sum of the selected events, where the weights were LASSO regression coefficients. The scoring system was applied to predict OS and time to leukemic change (TTLC) (flowchart in Supplementary Figure S2). With further analysis using the Geneious version 10.2.2 software (Biomatters, New Zealand), we could predict the 
consequence of protein changes by aberrant AS events among these aberrant AS genes.

\section{Validation of the array data}

Direct PCR followed by TA cloning with Taq polymerase-amplified (TA)-cloning vector pGEM $^{\circledast}-\mathrm{T}$ Easy (Promega, Madison, WI, USA) and sequencing or real time (RT)-PCR were performed to validate aberrant AS events detected by Affymetrix HTA 2.0. We used cDNA samples generated from total RNA extracted from BMMNCs of randomly selected MDS patients for validation. We chose the genes with high SI values and similar expression levels between the MDS patients and normal control donors for validation. AUP1 gene fulfilling the criteria was randomly chosen for further validation. Besides, another 2 genes PVRL2 and GRIK5 in LASSOCox regression model were also randomly picked for validation. GAPDH was selected as the internal control gene.

All the aberrant splicing transcripts were verified using cDNA of BMMNCs in 4 MDS patients and 3 normal control donors. AUP1 transcript was validated through PCR followed by TA cloning and sequencing. PVRL2 and GRIK5 were validated by RT-PCR method. The primer pairs used were shown below:

AUP1: forward (5'-AAGGAAGTTTTGCCCCATGT$\left.3^{\prime}\right)$ at exon 9

reverse $\left(5^{\prime}\right.$-CCCCCTCAAGCAGATTAGTG- $\left.3^{\prime}\right)$ at exon 10

PVRL2: forward (5'-CCCTCCTGAAGTGTCCATCT$\left.3^{\prime}\right)$ at exon 3

reverse (5'-TGCTCCAGTCATAGCCCGTG-3') at exon 4

GRIK5: forward (5'-TTCGTGGCGGTCATGGAATT$\left.3^{\prime}\right)$ at exon 18

reverse (5'-TTGCTGAGGCGCATCTCG-3') at exon 19

Due to different gene expression levels of GRIK5 between MDS patients and normal donors (fold change 2.9) at array raw data, verification of overall GRIK5 gene expression was performed using another primer pair $\left(5^{\prime}-\right.$ CCGGGATGAGATCACACTGG-3') and (5'-TGACCT CCGTGTGGACCATA-3') covering exon 17 and exon 18.

\section{Statistical analysis}

All statistical analyses were performed by SPSS version 17.0 software (SPSS Inc., Chicago, IL, USA). The ANOVA test was used for comparison of aberrant AS scores among different MDS subgroups based on FrenchAmerican-British (FAB) or World Health Organization (WHO) classification or genetic mutations. The Pearson correlation analysis was applied for the test of correlation between global aberrant AS scores with clinical and biological data. The Kaplan-Meier method was used to evaluate median OS and TTLC. OS was estimated from the date of first MDS diagnosis to the date of last followup or death due to any cause, and TTLC was measured as the duration from the date of initial MDS diagnosis to the date of acute leukemic change. Log-rank test was used to compare median OS and TTLC among different groups according to global aberrant AS scores and LASSO-Cox regression model. Univariate and multivariate Cox proportional hazard regression analysis were used to investigate independent prognostic factors for OS and TTLC. A $P$-value $<0.05$ was considered statistically significant.

\section{Results}

\section{Clinical characteristics of the patients}

Among the 176 MDS patients recruited in this study, most $(84.1 \%)$ received only supportive care. Four patients (2.3\%), two patients (1.1\%), and 22 patients (12.5\%) received intensive chemotherapy, hypomethylating agents, and allogeneic HSCT, respectively. The baseline characteristics of these patients were summarized in Table 1.

\section{Aberrant splicing of whole transcriptome}

This Affymetrix HTA 2.0 contains over 6 million distinct probes covering transcripts of 44,710 coding genes, 22,829 non-coding genes, and spanning the exon-exon junctions (ten probes per exon; four probes per exonexon junction). As a whole, there are 670,402 PSRs and 339,146 splice junction probe sets among 378,502 exons in 67,539 genes for evaluation by this array platform. This comprehensive coverage of the exons and junctions enables us to simultaneously determine gene expression levels and splicing patterns of every gene.

Totally 52,646 of 67,539 (77.9\%) genes on the array were expressed in BMMNCs from both MDS patients and normal controls. Approximately 26.9\% (14,162 of the $52,646)$ of the expressed genes were aberrantly spliced in the MDS patients, that is, these genes showed at least one aberrant AS event, compared with normal donors. Besides, a total of 42,760 aberrant AS events among these 14,162 aberrantly spliced genes were noted in the whole transcriptome. In addition, $85.9 \%$ of these aberrant splicing events were mapped to coding genome and $14.1 \%$ to non-coding regions.

\section{Associations of clinical features with aberrant splicing}

We calculated the aberrant AS score (i.e. total aberrant AS events divided by total aberrant AS genes) of each MDS patient with the 20 normal donors as reference. Our data displayed that aberrant AS scores were strongly correlated with total aberrant AS events among MDS patients $(r=0.964, P<0.001$, Fig. 1$)$. Therefore, a higher aberrant AS score not only indicated more aberrant AS 
Table 1 Baseline characteristics of 176 patients with primary MDS

\begin{tabular}{ll}
\hline Characteristics & Total number \\
\hline Age (ranged from 19 to 94 years, median 69 years) & \\
$\geqq 65$ years & $102(58 \%)$ \\
$<65$ years & $74(42 \%)$ \\
Gender & \\
Male & $121(69 \%)$ \\
Female & $55(31 \%)$ \\
FAB & \\
RA & $78(44 \%)$ \\
RARS & $22(13 \%)$ \\
RAEB & $76(43 \%)$ \\
WHO 2016 & \\
MDS-SLD & $40(23 \%)$ \\
MDS-MLD & $38(22 \%)$ \\
MDS-RS-SLD & $13(7 \%)$ \\
MDS-RS-MLD & $9(5 \%)$ \\
MDS-EB1 & $32(18 \%)$ \\
MDS-EB2 & $44(25 \%)$ \\
IPSS- (only 164 patients could be evaluated) & \\
Very low & $28(17 \%)$ \\
Low & $54(33 \%)$ \\
Intermediate & $41(25 \%)$ \\
\hline Very high & \\
\hline
\end{tabular}

FAB French-American-British classification, WHO World Health Organization, IPSS- $R$ revised international prognostic scoring system, MDS myelodysplastic syndrome, SLD single lineage dysplasia, MLD multilineage dysplasia, $R S$ ring sideroblasts, $E B$ excess blasts

events in each aberrant AS gene on average, but also reflected more aberrant AS events in the whole transcriptome and would imply a higher degree of aberrancy of AS. We calculated the aberrant AS score of each normal control donor with the other 19 donors as reference. The score among the 20 normal donors ranged from 1.25 to 3.21 with a median of 1.525 (Fig. 2a). In contrast, the aberrant AS scores ranged from 2.05 to 5.48 with a median of 2.60 in MDS patients (Fig. 2a) indicating significantly higher aberrant AS scores among MDS patients than normal donors $(P<0.001)$. We used receiver operating characteristic (ROC) curve for survival prediction of MDS patients to obtain a cut-off point value at 2.45 , which best differentiated the patients' survival. We defined a low and high global aberrant AS score by this value.

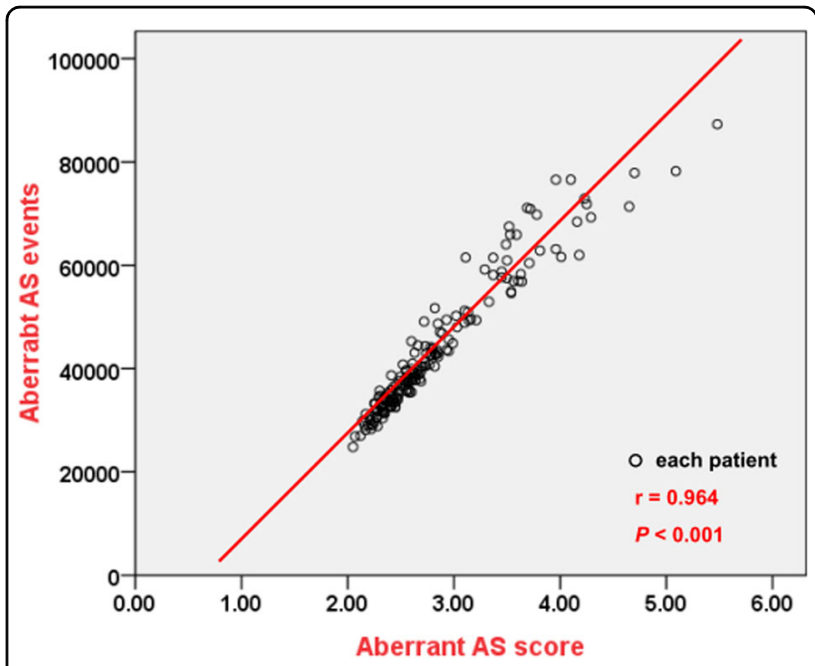

Fig. 1 Aberrant AS scores and total aberrant AS events among MDS patients. Aberrant AS scores were strongly correlated with total aberrant AS events of whole transcriptome among 176 MDS patients

A higher global aberrant AS score was significantly associated with male gender and U2AF1 mutation (Table 2). But the aberrant AS scores were not significantly different among different MDS subgroups according to the FAB or $2016 \mathrm{WHO}$ classification (Fig. 2b, c). They were also not significantly different among the patients classified by the status of mutations in genes related to spliceosome and epigenetic modifications (Fig. 2d, Supplementary Figure S3). There was no association between aberrant AS scores and age, hemogram, or cytogenetics, either (Table 2).

\section{The prognostic impact of aberrant AS scores}

With a median follow up time of 48.4 months, a higher global aberrant AS score, indicating a higher degree of genome-wide aberrant AS pattern, predicted significantly shorter overall survival (OS) (median, 21.7 vs. 69.9 months, $P=0.009$ ) and shorter time to leukemic change (TTLC) (first quartile, 12.5 months vs. not reached, $P=0.002$ ) than those with a lower score (Fig. 3a, b). In order to clarify the pathological effects of aberrant splicing in the absence of mutations in either spliceosome complex or epigenetic modifiers, which were common in MDS patients ${ }^{22}$, we focused on subgroups of MDS patients without detectable mutations in genes related to spliceosome or epigenetic modifications and found that a higher global aberrant AS score could also predict shorter OS in these patients (Fig. 3c). Moreover, we were able to confirm that a higher global aberrant AS score could predict shorter OS in the validation cohort of 31 primary MDS patients (Fig. 3d).

Multivariate analysis including variables that had prognostic significance in univariate analysis 

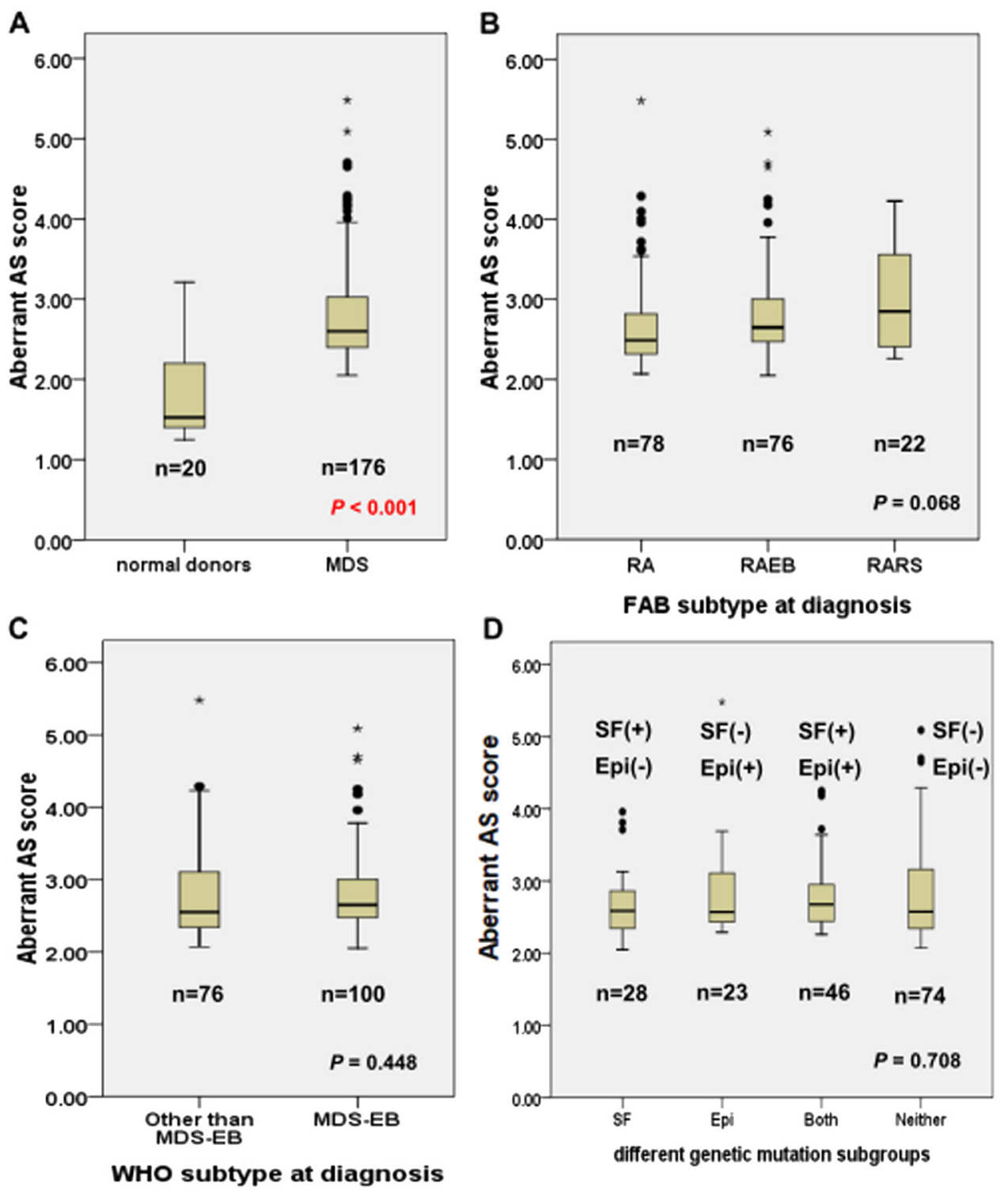

Fig. 2 Aberrant AS scores among MDS patients. Aberrant AS scores in total cohort of 176 MDS patients and 20 normal donors (a), different MDS subgroups according to the FAB classification (b), 2016 WHO classification (c), and mutation status in splicing factor (SF) genes and epigenetic (Epi) genes (d). SF genes include four genes: SF3B1, U2AF1, SRSF2, and ZRSR2. Epigenetic genes include six genes: TET2, ASXL1, DNMT3A, EZH2, IDH1 and IDH2. SF(+): at least one splicing factor gene mutation; Epi(+): at least one epigenetic gene mutation; SF(-): no splicing factor gene mutation; Epi(-): no epigenetic gene mutation; • Dots: mild outlier; * Stars: extreme outlier; bold horizontal lines: median

(Supplementary Table S1) in the 176 MDS patients showed that a high global aberrant AS score was an independent unfavorable prognostic factor for both OS and TTLC irrespective of age, revised international prognostic scoring system (IPSS-R) risk, and mutations in SRSF2, ZRSR2, ASXL1, TP53, and EZH2 (Table 3).

Tefferi et al. recently developed a new prognostic model for primary MDS patients by integration of genetic and clinical information ${ }^{23}$. This Mayo Alliance Prognostic Model provides another platform for prognostication in MDS patients other than the IPSS-R. We were interested if the prognostic significance of aberrant splicing might hold up in the context of this new scoring system. We found that a higher degree of global aberrant AS was still an independent poor risk factor after adjustment for Mayo Alliance model by Cox regression analysis (Supplementary Table S2). 
Table 2 Comparison of clinical manifestations between MDS patients with high and low global aberrant alternative splicing (AS) score

\begin{tabular}{|c|c|c|c|c|}
\hline Variables & Total & $\begin{array}{l}\text { High } \\
\text { aberrant AS } \\
\text { score }\end{array}$ & $\begin{array}{l}\text { Low aberrant } \\
\text { AS score }\end{array}$ & $P$ value \\
\hline & $(n)$ & $(n)$ & $(n)$ & \\
\hline $\operatorname{sex}^{a}$ & & & & $0.024^{*}$ \\
\hline Male & 121 & 87 & 34 & \\
\hline Female & 55 & 30 & 25 & \\
\hline Age $(\text { year })^{b}$ & 69 (19-94) & $66(19-89)$ & $73(26-94)$ & 0.121 \\
\hline$\geqq 65$ & 102 & 63 & 39 & \\
\hline$<65$ & 74 & 54 & 20 & \\
\hline \multicolumn{5}{|c|}{ Laboratory data $^{\mathrm{b}}$} \\
\hline WBC $(/ \mu \mathrm{L})$ & $\begin{array}{l}3825 \\
(490-20440)\end{array}$ & $\begin{array}{l}3780 \\
(490-20440)\end{array}$ & $\begin{array}{l}4660 \\
(1710-11690)\end{array}$ & 0.426 \\
\hline $\mathrm{Hb}(\mathrm{g} / \mathrm{dL})$ & $8.1(3.5-14.6)$ & $8.1(3.5-13.6)$ & $8.1(3.7-14.6)$ & 0.792 \\
\hline $\begin{array}{l}\text { Platelet } \\
(\times 1000 / \mu \mathrm{L})\end{array}$ & $85(3-721)$ & $82(9-721)$ & $106(3-460)$ & 0.08 \\
\hline \multicolumn{5}{|l|}{ Cytogenetics $^{a}$} \\
\hline Favorable $^{c}$ & 115 & 71 & 44 & 0.09 \\
\hline Intermediate ${ }^{d}$ & 23 & 17 & 6 & 0.382 \\
\hline Poor $^{e}$ & 26 & 20 & 6 & 0.197 \\
\hline \multicolumn{5}{|c|}{ Genetic alterations $^{\mathrm{a}}$} \\
\hline SF3B1 & 29 & 17 & 12 & 0.342 \\
\hline U2AF1 & 14 & 13 & 1 & $0.028^{*}$ \\
\hline SRSF2 & 24 & 15 & 9 & 0.675 \\
\hline ZRSR2 & 15 & 12 & 3 & 0.254 \\
\hline TET2 & 22 & 17 & 5 & 0.246 \\
\hline ASXL1 & 36 & 26 & 10 & 0.386 \\
\hline DNMT3A & 25 & 18 & 7 & 0.517 \\
\hline EZH2 & 10 & 7 & 3 & 0.809 \\
\hline IDH1/IDH2 & 4 & 4 & 0 & 0.151 \\
\hline TP53 & 12 & 10 & 2 & 0.193 \\
\hline RUNX1 & 25 & 18 & 7 & 0.517 \\
\hline
\end{tabular}

*Statistically significant $(P<0.05)$

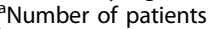

${ }^{\mathrm{b}}$ Median (range)

'Favorable cytogenetics: -Y, del(11q), Normal, del(5q), del(12p), del(20q), double including del $(5 q)$

IIntermediate-risk cytogenetics: del(7q), $+8,+19, \mathrm{i}(17 \mathrm{q})$, any other single or double independent clones

Poor-risk cytogenetics: -7 , inv(3)/t(3q)/del(3q), double including $-7 / \mathrm{de}(7 \mathrm{q})$, complex: 3 abnormalities, complex: $>3$ abnormalities

\section{Physiological significance of the aberrant splicing}

To explore the physiological pathways perturbed by the aberrant splicing, we analyzed the genes with aberrant AS by DAVID database. We identified 71 signaling pathways significantly affected by aberrant splicing with $P$ value $<$ 0.001 and false discovery rate $($ FDR $)<0.05$. We listed the top 20 pathways according to fold enrichment and discovered that pathways related to cell proliferation, adhesion, and protein degradation were affected by the aberrant AS (Supplementary Table S3). These findings indicated that the normal splicing mechanism was disrupted in MDS patients and the aberrant splicing in signaling networks seemed to be widespread which perturbed many normal physiological pathways.

\section{Aberrant AS events with prognostic significance}

We then sought to identify the core subsets of aberrant AS events that could predict the prognosis of MDS patients. A univariate Cox analysis identified 53 OSassociated aberrant AS events (Bonferroni adjusted $P<$ 0.05). Among them, a subset of 13 aberrant AS events could best predict OS by a LASSO-Cox regression analysis. As a result, we developed a simple scoring system composed of a weighted sum of the SI of the 13 aberrant AS events on ARHGEF17, C1QTNF4, EGFL7, GNAI1, GRIK5, HOXA9, KRT18, MAP3K15, MEG3, PTK7, PVRL2, PXDN, and TGFBI (Supplementary Table S4). The scores of LASSO-Cox regression model ranged from -0.5 to 3.53 in our discovery cohort. With a cut-off point at the median value 1.14, a high score was significantly predictive of inferior OS (Fig. 4a; $P<0.001$ ) and shorter TTLC (Fig. 4b; $P<0.001$ ). The result was confirmed in the validation cohort (Fig. 4c; $P<0.001$ ).

\section{Validation of the array-predicted splicing}

To verify the aberrant AS events identified by the array, we chose 3 gene transcripts for validation as mentioned in Materials and Methods. AUP1 gene is located at 2P13.1 and contains 12 exons; the transcript (NM_181575) showed aberrant retention of intron 9 (mean SI level 3.54) among most MDS patients (71.6\%) in our cohort at HTA 2.0. An obvious additional band of AUP1 specific transcripts at 204 base pairs was present in the 4 MDS patients studied, compared with 3 normal control donors (Fig. 5a). We verified the retention of intron 9 in AUP1 in these MDS patients by direct sequencing (Fig. $5 \mathrm{~b}-\mathrm{d}$ ). The aberrant AS of AUP1 in MDS patients would lead to protein truncation by the prediction of Geneious software (Supplementary Figure S4). PVRL2 gene (NM_001042724) is located at 19q13.32 and contains 9 exons; the array data showed exon 4 skipping in almost all of our MDS patients (98.3\%). GRIK5 gene (NM_002088) is located at $19 \mathrm{q} 13.2$ and contains 19 exons; the array data showed exon 19 skipping in most of our MDS patients (84.1\%). By RT-PCR method, we verified exon 4 skipping in PVRL2 gene and exon 19 skipping in GRIK5 gene in the 4 MDS patients analyzed (Fig. 5e, f). 

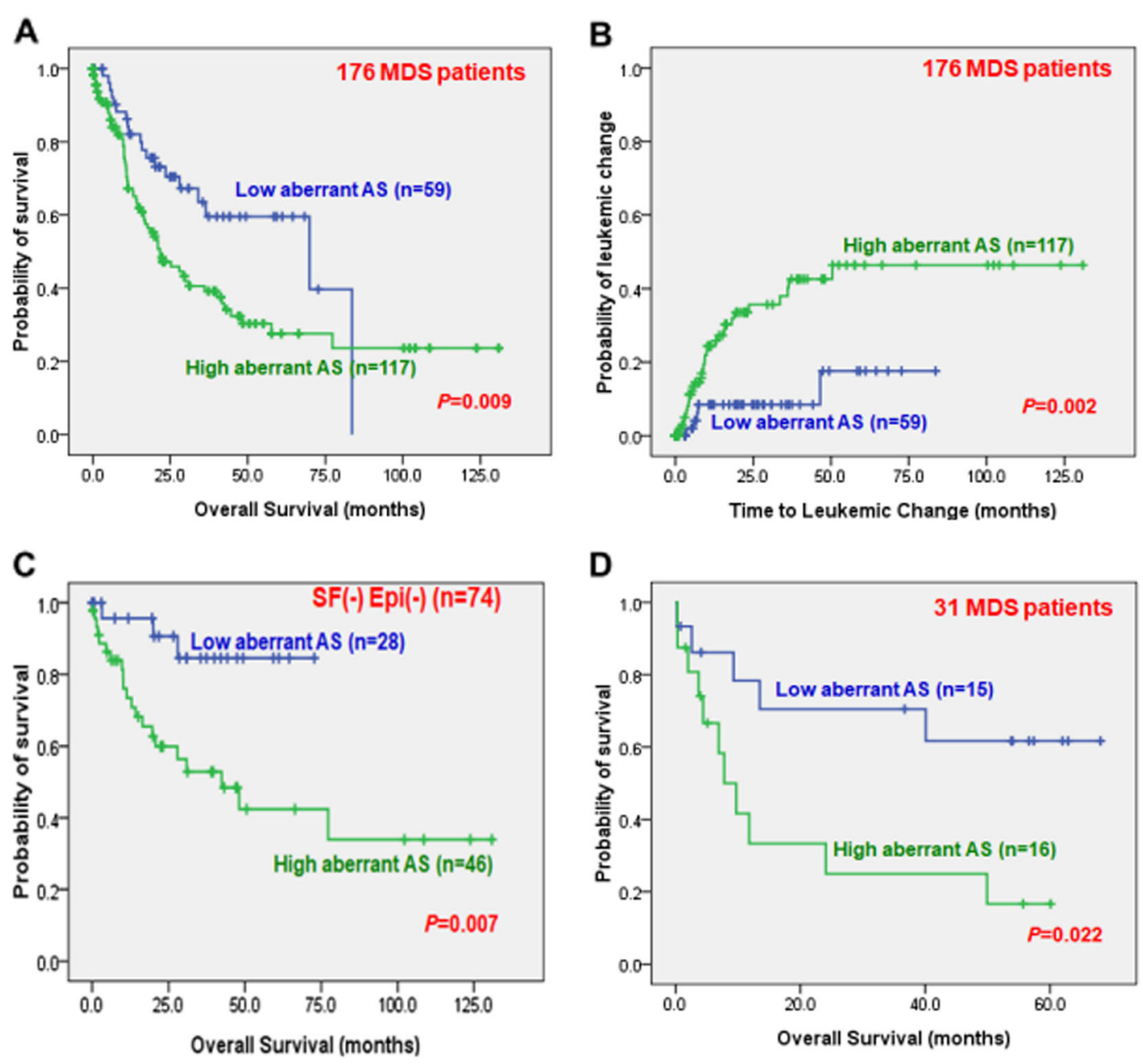

Fig. 3 Prognostic impact of genome-wide aberrant AS pattern among MDS patients. Kaplan-Meier survival curves stratified by genome-wide aberrant AS pattern (with a cut-off point value at 2.45 of aberrant AS score) for overall survival (OS) (a) and time to leukemic change in total cohort of 176 MDS patients (b), OS in patients without any detectable mutation in genes related to splicing factors or epigenetic modifications (c), and OS in the validation cohort of 31 primary MDS patients (d). Note: SF(-)Epi(-) means neither splicing factor, nor epigenetic genes mutation

\section{Discussion}

Alternative splicing is crucial for normal hematopoiesis; disruption of this physiological process may be related to leukemogenesis ${ }^{24}$. Global aberrant splicing has been reported in many diseases including benign and malignant disorders, such as cystic fibrosis, Alzheimer disease and many cancers, as well as $\mathrm{AML}^{2,5,6,25,26}$. Some splicing variants of specific genes have been proposed to serve as diagnostic or prognostic biomarkers for cancer patients $^{27,28}$. Although aberrant AS of specific genes has been reported in MDS, the global pattern of splicing in this disease has not been well explored ${ }^{29-32}$.

Previous studies used expressed sequence tags (ESTs) to identify aberrant AS events ${ }^{33}$, but this method was limited by small numbers of ESTs. Exon array is another tool but lacks comprehensive coverage at the exon-exon junctions ${ }^{25}$. Recently, next generation sequencing-based RNA- seq has been widely used for investigation of alternative splicing, but deep reads are necessary for this purpose ${ }^{1,34}$. One recent study compares Illumina HiSeq 2000 with HTA 2.0 for detection of alternative splicing and concludes that modern microarrays outperform sequencing for standard analysis of gene expression in terms of reproducibility and $\operatorname{cost}^{35}$.

In this study, we used BMMNCs rather than a more homogenous population such as sored $\mathrm{CD} 34^{+}$cells. A concern is the possible heterogeneity of gene expression profiles and splicing patterns in BMMNCs ${ }^{36,37}$. Nevertheless, in a recent report regarding molecular classification based on gene expression and its correlation with survival and risk of leukemic transformation in MDS, Shiozawa et al. found that the prognostic value of the classification according to the gene expression profiles of BMMNCs was similar to that of the purified CD34 
Table 3 Multivariate analysis (Cox regression) on the overall survival and time to leukemic change

\begin{tabular}{|c|c|c|c|c|c|c|c|c|}
\hline \multirow[t]{3}{*}{ Variables } & \multicolumn{4}{|c|}{ Overall survival } & \multicolumn{4}{|c|}{ Time to leukemic change } \\
\hline & \multirow[t]{2}{*}{ HR } & \multicolumn{2}{|l|}{$95 \% \mathrm{Cl}$} & \multirow[t]{2}{*}{$P$} & \multirow[t]{2}{*}{$H R$} & \multicolumn{2}{|l|}{$95 \% \mathrm{Cl}$} & \multirow[t]{2}{*}{$P$} \\
\hline & & Lower & Upper & & & Lower & Upper & \\
\hline $\mathrm{Age}^{\mathrm{a}}$ & 1.019 & 1.003 & 1.035 & $0.017^{*}$ & 0.984 & 0.965 & 1.005 & 0.133 \\
\hline IPSS-R ${ }^{b}$ & 3.114 & 1.839 & 5.270 & $<0.001^{*}$ & 3.694 & 1.710 & 7.982 & $0.001^{*}$ \\
\hline SRSF2 & 1.092 & 0.501 & 2.379 & 0.825 & 0.913 & 0.294 & 2.837 & 0.875 \\
\hline ZRSR2 & 1.400 & 0.665 & 2.950 & 0.376 & 0.690 & 0.212 & 2.242 & 0.537 \\
\hline ASXL1 & 1.628 & 0.784 & 3.381 & 0.191 & 4.788 & 1.933 & 11.857 & $0.001^{*}$ \\
\hline$E Z H 2$ & 1.227 & 0.456 & 3.300 & 0.685 & 0.701 & 0.171 & 2.881 & 0.622 \\
\hline TP53 & 4.563 & 1.936 & 10.753 & $0.001^{*}$ & 5.009 & 1.250 & 20.079 & $0.023^{*}$ \\
\hline Aberrant AS score ${ }^{c}$ & 1.801 & 1.019 & 3.181 & $0.043^{*}$ & 2.736 & 1.036 & 7.222 & $0.042^{*}$ \\
\hline
\end{tabular}

$H R$ hazard ratio, $\mathrm{Cl}$ confidence interval

* Statistically significant $(P<0.05)$

${ }^{a}$ Age as a continuous variable

IIPSS-R risk score $>4.5$ relative to IPSS-R $\leq 4.5$ (the reference)

'High global aberrant AS score relative to low global aberrant AS score (the reference)
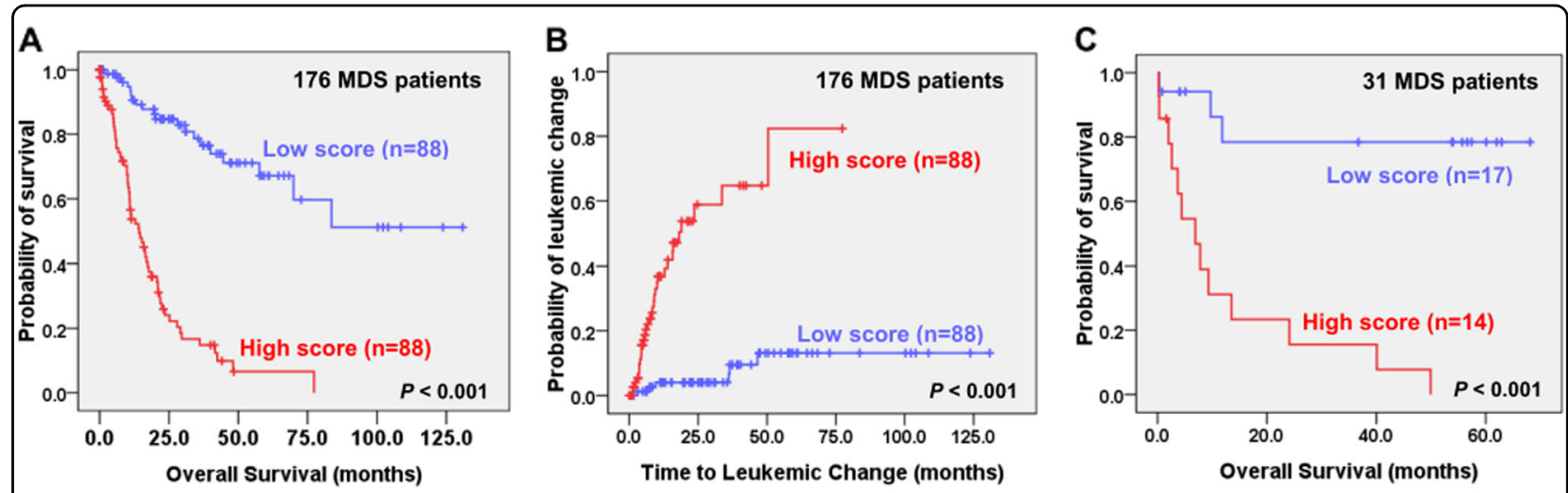

Fig. 4 Prognostic impact of Lasso-Cox regression model among MDS patients. Kaplan-Meier survival curves stratified by the scoring system of LASSO-Cox regression model composed of 13 aberrant AS events for OS (a) and time to leukemic change (b) in total cohort of 176 primary MDS patients, and OS in validation cohort of 31 primary MDS patients (c)

progenitor cells ${ }^{38}$. Further studies are warranted to clarify this concern.

To the best of our knowledge, this is the first report regarding the clinical implications of global splicing aberrancies in MDS patients. By a new platform of arrays containing extensive exon and exon-exon junctional probes, we were able to investigate the genome-wide splicing patterns. We found drastic differences in genome-wide splicing patterns between MDS patients and healthy donors.

Our data revealed $26.9 \%$ of expressed genes with aberrant splicing in MDS patients compared to normal BM donors. In general, there were 42,760 aberrant AS events in whole MDS patients (ranged from 24,829 to 87,286 events of each MDS patient) noted at the transcriptome by our HTA 2.0 platform. By contrast, some previous studies with RNA-seq platform reported about 1000 to 125000 aberrant AS events in about 290000 splice junctions examined ${ }^{39,40}$. The reason that the number of aberrant AS events differs among different studies might be explained by different patient populations and study platforms. In addition, these MDS-specific splicing aberrancies were located in both the coding and non-coding regions, as reported by others ${ }^{5}$. It is likely that the aberrant splicing in MDS would lead to alteration of expression in many genes, affect many important cellular processes, and contribute at least partially to the development of this disease. 


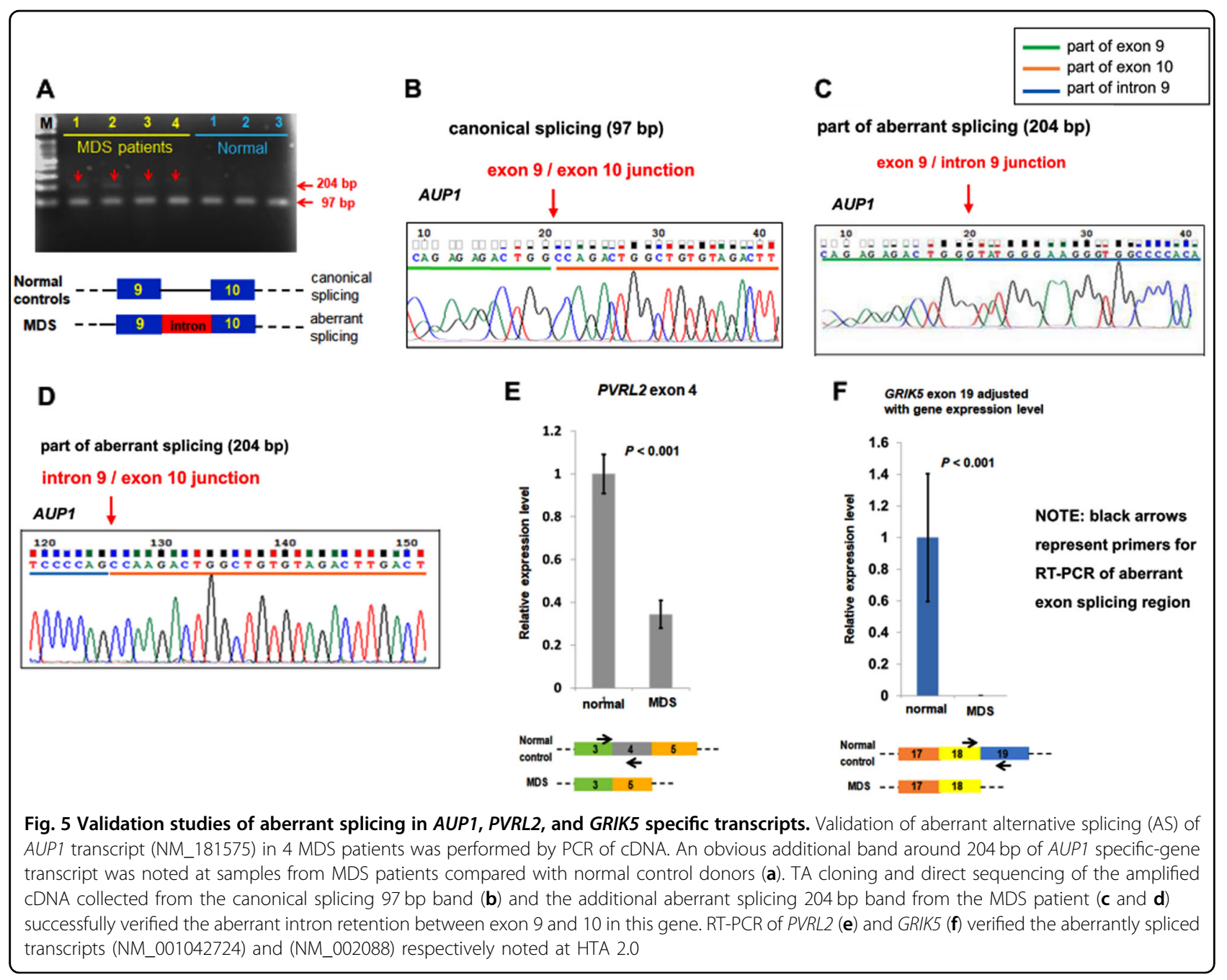

We quantified the aberrant splicing by "aberrant AS score" defined as total aberrant AS events divided by total aberrant AS genes in each MDS patient. A higher aberrant AS score could reflect a higher degree of genome-wide aberrancies of AS pattern as shown in Fig. 1. With this parameter, we noted the extent of aberrant splicing could predict OS and TTLC in MDS patients, independent of other well established prognostic factors such as age, IPSS-R and important genetic mutations, although it was not different among the risk groups of patients nor was it affected by genetic mutation categories. In a previous study regarding aberrant splicing in AML, CD13 splice variants were reduced in remission but dramatically increased at relapse $\mathrm{s}^{5}$. However, the prognostic implication of aberrant AS was not studied in that report. In our study, we found a higher degree of aberrancies of AS predicted a poor outcome. Interestingly, a high global aberrant AS score was only significantly associated with $U 2 A F 1$ mutation $^{41}$. It is likely that many factors other than gene mutations involving spliceosome or epigenetic modifiers are responsible for the aberrant splicing.

While the degree of AS aberrancies harbored prognostic significance, we were interested in finding specific aberrant AS genes with prognostic significance. These genes may have implications for the pathogenesis of MDS. To this end, we performed a LASSO-Cox regression model to analyze the power of OS prediction among all aberrant AS events and identified 13 events with prominent prognostic significance. The scoring system composed of these 13 specific aberrant AS events could predict both OS and TTLC in MDS patients. These aberrant splicing events might lead to protein loss (nonsense-mediated mRNA decay rule), protein truncation, in-frame peptide insertion/deletion. In addition, alterations in untranslated regions or non-coding RNA are likely to perturb normal gene functions. Among the genes affected, four (C1QTNF4, EGFL7, MAP3K15, PTK7) may affect cell proliferation signaling pathway ${ }^{42-45}$; four (KRT18, PVRL2, 
PXDN, TGFBI) can alter cell adhesion leading to inhibition of apoptosis and increasing tumor invasiveness ${ }^{46-49}$; two genes (ARHGEF17, GNAI1) are supposed to regulate apoptotic pathway ${ }^{50,51}$; HOXA9 is a transcription factor and MEG3 is a tumor suppressor gene ${ }^{52,53}$. The last gene GRIK5 forms functional heteromeric kainate-preferring ionic channels while its biological implication for carcinogenesis is unknown yet ${ }^{54}$. Further studies are needed to verify the biological significances of these genes in the pathogenesis of MDS.

In summary, for the first time, our data clearly demonstrated that the complexity of global aberrant splicing had prognostic impacts on MDS patients. Studies in large prospective cohorts are needed to confirm our observations.

\section{Acknowledgements}

We thank Sue-Wei Nein of National Health Research Institutes Microarray Core Lab for the management of HTA 2.0. The study was supported by grants from National Taiwan University Hospital-National Taiwan University joint research grant (UN103-051), Ministry of Science and Technology of Taiwan (MOST1022325-B-002-028, 103-2314-B-002-130-MY3, 103-2314-B-002-131MY3, 104-2923B-002-001, 106-2314-B-002-224-MY3 and 106-2314-B-002-231), Far Eastern Hospital and NTUH joint grant 105-FTN24, Ministry of Health and Welfare of Taiwan (MOHW106-TDU-B-211-144005) from the National Science Council (Taiwan), MOHW103-TD-B-111-04 from the Ministry of Health and Welfare (Taiwan), 107-HCH026 from the Department of Medical Research, National Taiwan University Hospital Hsin-Chu Branch and NTUH $102 P 06$ and UN 102-015 from the Department of Medical Research, National Taiwan University Hospital.

\section{Author details}

'Division of Hematology-Oncology, Department of Internal Medicine, National Taiwan University Hospital Hsin-Chu Branch, Hsinchu, Taiwan. ${ }^{2}$ Graduate Institute of Clinical Medicine, College of Medicine, National Taiwan University, Taipei, Taiwan. ${ }^{3}$ Greehey Children's Cancer Research Institute, UT Health San Antonio, San Antonio, TX, USA. ${ }^{4}$ Division of Hematology, Department of Internal Medicine, National Taiwan University Hospital, Taipei, Taiwan. ${ }^{5}$ Laboratory Medicine, National Taiwan University Hospital, Taipei, Taiwan. ${ }^{6}$ TaiChang Stem Cell Therapy Center, National Taiwan University, Taipei, Taiwan

\section{Authors contributions}

Y.-T.Y. was responsible for literature collection, data management and interpretation, and manuscript writing; Y.-C.C. was responsible for statistical analysis, interpretation of the statistical findings and manuscript writing; C.J.K., was responsible for RT-PCR, TA cloning and sequencing; M.H.T. performed the gene mutation and chromosomal studies; H.-A.H., C.-C.L., C.-H.T., contributed patient samples and clinical data collection; W.-C.C. and H.-F.T. planned, designed, wrote manuscript and coordinated the study over the entire period.

\footnotetext{
Author details

${ }^{7}$ Division of Hematology-Oncology, Department of Internal Medicine, National Taiwan University Hospital Hsin-Chu Branch, Hsinchu, Taiwan. ${ }^{8}$ Graduate Institute of Clinical Medicine, College of Medicine, National Taiwan University, Taipei, Taiwan. ${ }^{9}$ Greehey Children's Cancer Research Institute, UT Health San Antonio, San Antonio, TX, USA. ${ }^{10}$ Division of Hematology, Department of Internal Medicine, National Taiwan University Hospital, Taipei, Taiwan.

${ }^{11}$ Laboratory Medicine, National Taiwan University Hospital, Taipei, Taiwan.

${ }^{12}$ Tai-Chang Stem Cell Therapy Center, National Taiwan University, Taipei, Taiwan
}

Conflict of interest

The authors declare that they have no conflict of interest.

\section{Publisher's note}

Springer Nature remains neutral with regard to jurisdictional claims in published maps and institutional affiliations.

Supplementary Information accompanies this paper at (https://doi.org/ 10.1038/s41408-018-0115-2).

Received: 19 May 2018 Revised: 11 July 2018 Accepted: 18 July 2018 Published online: 13 August 2018

\section{References}

1. Pan, Q., Shai, O., Lee, L. J., Frey, B. J. \& Blencowe, B. J. Deep surveying of alternative splicing complexity in the human transcriptome by highthroughput sequencing. Nat. Genet. 40, 1413-1415 (2008).

2. Srebrow, A. \& Kornblihtt, A. R. The connection between splicing and cancer. J. Cell Sci. 119, 2635-2641 (2006).

3. Blencowe, B. J. Alternative splicing: new insights from global analyses. Cell 126, $37-47$ (2006).

4. Sveen, A., Kilpinen, S., Ruusulehto, A., Lothe, R. A. \& Skotheim, R. I. Aberrant RNA splicing in cancer; expression changes and driver mutations of splicing factor genes. Oncogene 35, 2413-2427 (2016).

5. Adamia, S. et al. A genome-wide aberrant RNA splicing in patients with acute myeloid leukemia identifies novel potential disease markers and therapeutic targets. Clin. Cancer Res. 20, 1135-1145 (2014).

6. Zhou, J. \& Chng, W. J. Aberrant RNA splicing and mutations in spliceosome complex in acute myeloid leukemia. Stem Cell Investig. 4, 6 (2017).

7. Arber, D. A. et al. The2016 revision to the World Health Organization classification of myeloid neoplasms and acute leukemia. Blood 127, 2391-2405 (2016).

8. Shaffer, L. GSlovak, M. LCampbell, L. J. (eds). ISCN 2009: An International System for Human Cytogenetic Nomenclature. (S. Karger, Basel, 2009).

9. Lin, C. C. et al. SF3B1 mutations in patients with myelodysplastic syndromes: the mutation is stable during disease evolution. Am. J. Hematol. 89, E109-E115 (2014).

10. Hou, H. A. et al. Splicing factor mutations predict poor prognosis in patients with de novo acute myeloid leukemia. Oncotarget 7, 9084-9101 (2016).

11. Wu, S. J. et al. The clinical implication of SRSF2 mutation in patients with myelodysplastic syndrome and its stability during disease evolution. Blood 120, 3106-3111 (2012).

12. Chou, W. C. et al. TET2 mutation is an unfavorable prognostic factor in acute myeloid leukemia patients with intermediate-risk cytogenetics. Blood 118, 3803-3810 (2011).

13. Chen, T. C. et al. Dynamics of ASXL1 mutation and other associated genetic alterations during disease progression in patients with primary myelodysplastic syndrome. Blood Cancer J. 4, e177 (2014).

14. Hou, H. A. et al. DNMT3A mutations in acute myeloid leukemia: stability during disease evolution and clinical implications. Blood 119, 559-568 (2012).

15. Chou, W. C. et al. Distinct clinical and biologic characteristics in adult acute myeloid leukemia bearing the isocitrate dehydrogenase 1 mutation. Blood 115, 2749-2754 (2010).

16. Lin, C. C. et al. IDH mutations are closely associated with mutations of DNMT3A, ASXL1 and SRSF2 in patients with myelodysplastic syndromes and are stable during disease evolution. Am. J. Hematol. 89, 137-144 (2014).

17. Yao, C. Y. et al. Distinct mutation profile and prognostic relevance in patients with hypoplastic myelodysplastic syndromes (h-MDS). Oncotarget 7, 63177-63188 (2016)

18. Hou, H. A. et al. Incorporation of mutations in five genes in the revised International Prognostic Scoring System can improve risk stratification in the patients with myelodysplastic syndrome. Blood Cancer J. 8, 39 (2018).

19. Clark, T. A., Sugnet, C. W. \& Ares, M. Jr. Genomewide analysis of mRNA processing in yeast using splicing-specific microarrays. Science 296, 907-910 (2002).

20. Srinivasan, $\mathrm{K}$. et al. Detection and measurement of alternative splicing using splicing-sensitive microarrays. Methods 37, 345-359 (2005).

21. Qian J., Hastie T., Friedman J., Tibshirani R., Simon N. GImnet for Matlab; 2013. https://web.stanford.edu/ hastie/glmnet_matlab/

22. Bejar, R. \& Steensma, D. P. Recent developments in myelodysplastic syndromes. Blood 124, 2793-2803 (2014). 
23. Tefferi A., et al. Mayo Alliance Prognostic Model for Myelodysplastic Syndromes: Integration of Genetic and Clinical Information. Mayo Clin Proc 2018; https://doi.org/10.1016/j.mayocp.2018.04.013.

24. Pritsker, M. et al. Diversification of stem cell molecular repertoire by alternative splicing. Proc. Natl Acad. Sci. USA 102, 14290-14295 (2005).

25. Thorsen, K. et al. Alternative splicing in colon, bladder, and prostate cancer identified by exon array analysis. Mol. Cell. Proteom. 7, 1214-1224 (2008).

26. Garcia-Blanco, M. A., BaraniakA. P., \& LasdaE. L. Alternative splicing in disease and therapy. Nat. Biotechnol. 22, 535-546 (2004).

27. Brinkman, B. M. Splice variants as cancer biomarkers. Clin. Biochem. 37 584-594 (2004).

28. Omenn, G. S., Yocum, A. K. \& Menon, R. Alternative splice variants, a new class of protein cancer biomarker candidates: findings in pancreatic cancer and breast cancer with systems biology implications. Dis. Markers 28, 241-251 (2010).

29. Maratheftis $\mathrm{Cl}$ et al. Aberrant alternative splicing of interferon regulatory factor1 (IRF-1) in myelodysplastic hematopoietic progenitor cells. Leuk. Res. 30, 1177-1186 (2006)

30. Caudill, J. S., Porcher, J. C. \& Steensma, D. P. Aberrant pre-mRNA splicing of a highly conserved cell cycle regulator, CDC25C, in myelodysplastic syndromes. Leuk. Lymphoma 49, 989-993 (2008).

31. Dolatshad, H. et al. Cryptic splicing events in the iron transporter ABCB7 and other key target genes in SF3B1-mutant myelodysplastic syndromes. Leukemia 30, 2322-2331 (2016)

32. Dong, W. et al. MDS shows a higher expression of hTERT and alternative splice variants in unactivated T-cells. Oncotarget 7, 71904-71914 (2016).

33. Adams, M. D. et al. Complementary DNA sequencing: expressed sequence tags and human genome project. Science 252, 1651-1656 (1991).

34. Raghavachari, N. et al. A systematic comparison and evaluation of high density exon arrays and RNA-seq technology used to unravel the peripheral blood transcriptome of sickle cell disease. BMC Med. Genom. 5, 28 (2012).

35. Nazarov, P. V. et al. RNA sequencing and transcriptome arrays analyses show opposing results for alternative splicing in patient derived samples. BMC Genom. 18, 443 (2017).

36. Walter, M. J. et al. Clonal architecture of secondary acute myeloid leukemia. $N$. Engl. J. Med. 366, 1090-1098 (2012).

37. Walter, M. J. et al. Clonal diversity of recurrently mutated genes in myelodysplastic syndromes. Leukemia 27, 1275-1282 (2013).

38. Shiozawa, $Y$. et al. Gene expression and risk of leukemic transformation in myelodysplasia. Blood 130, 2642-2653 (2017).
39. Madan, V. et al. Aberrant splicing of U12-type introns is the hallmark of ZRSR2 mutant myelodysplastic syndrome. Nat. Commun. 6, 6042 (2015).

40. Kim, E. et al. SRSF2 mutations contribute to myelodysplasia by mutant-specific effects on exon recognition. Cancer Cell 27, 617-630 (2015).

41. Przychodzen, B. et al. Patterns of missplicing due to somatic U2AF1 mutations in myeloid neoplasms. Blood 122, 999-1006 (2013).

42. Li, Q. et al. Identification of C1qTNF-related protein 4 as a potential cytokine that stimulates the STAT3 and NF-KB pathways and promotes cell survival in human cancer cells. Cancer Lett. 308, 203-214 (2011).

43. Nichol, D. \& Stuhlmann, H. EGFL7: a unique angiogenic signaling factor in vascular development and disease. Blood 119, 1345-1352 (2012).

44. Kaji, T. et al. ASK3, a novel member of the apoptosis signal-regulating kinase family, is essential for stress-induced cell death in HeLa cells. Biochem. Biophys. Res. Commun. 395, 213-218 (2010).

45. Puppo, F. et al. Protein tyrosine kinase 7 has a conserved role in Wnt/B-catenin canonical signalling. EMBO Rep. 12, 43-49 (2011).

46. Zhang, B. et al. Cytokeratin 18 knockdown decreases cell migration and increases chemosensitivity in non-small cell lung cancer. J. Cancer Res. Clin. Oncol. 142, 2479-2487 (2016).

47. Samanta, D. et al. Structure of Nectin-2 reveals determinants of homophilic and heterophilic interactions that control cell-cell adhesion. Proc. Natl Acad. Sci. USA 109, 14836-14840 (2012).

48. Péterfi, Z. et al. Peroxidasin is secreted and incorporated into the extracellular matrix of myofibroblasts and fibrotic kidney. Am. J. Pathol. 175, 725-735 (2009).

49. Thapa, N., Lee, B. H. \& Kim, I. S. TGFBlp/betaig-h3 protein: a versatile matrix molecule induced by TGF-beta. Int. J. Biochem. Cell. Biol. 39, 2183-2194 (2007).

50. Lutz, S., Mohl, M., Rauch, J., Weber, P. \& Wieland, T. RhoGEF17, a Rho-specific guanine nucleotide exchange factor activated by phosphorylation via cyclic GMP-dependent kinase la. Cell. Signal. 25, 630-638 (2013).

51. O'Hayre, M. et al. The emerging mutational landscape of $G$ proteins and $G$ protein-coupled receptors in cancer. Nat. Rev. Cancer 13, 412-424 (2013).

52. Argiropoulos, B. \& Humphries, R. K. Hox genes in hematopoiesis and leukemogenesis. Oncogene 26, 6766-6776 (2007).

53. He, C. et al. Long noncoding RNA MEG3 negatively regulates proliferation and angiogenesis in vascular endothelial cells. DNA Cell Biol. 36, 475-481 (2017).

54. Szpirer, $C$. et al. The genes encoding the glutamate receptor subunits KA1 and KA2 (GRIK4 and GRIK5) are located on separate chromosomes in human, mouse, and rat. Proc. Natl Acad. Sci. USA 91, 11849-11853 (1994). 